\title{
Biological Applications of Fluorescence Photoactivation Localization Microscopy
}

\author{
Kristin A. Gabor
}

Graduate School of Biomedical Sciences, University of Maine, Orono, ME 04469

Since the advent of super-resolution imaging methods, great advances have been made in our capability to understand biological systems. While diffraction limits the smallest structures that can be imaged in a conventional light microscope to approximately 200-250 nanometers, many biological processes occur on molecular length scales. Fluorescence photoactivation localization microscopy [1,2,3] (FPALM) can image biological samples with resolution of 20-30 nm. This recently developed method offers a means by which previously inaccessible biological questions can now be addressed.

In conventional fluorescence microscopy, the majority of labeled molecules are visible at the same time, resulting in diffraction-dependent blurring of structures on length scales less than 200-250 nm. In contrast, localization microscopy gathers image information by measuring the positions of (localizing) many small subsets of fluorescently labeled molecules within a sample. By imaging these molecules separately and stochastically, at a low enough density where each visible molecule is spatially distinct from the others, fluorescent molecules imaged with FPALM can be localized with a precision of just a few nanometers, and with an overall density of $>10000$ per $\mathrm{um}^{2}$ within the focal plane. Images are then rendered by plotting the positions of all localized molecules.

Localization microscopy has rapidly evolved to image live cells [1], multiple molecular species [4,5], three-dimensional samples [6,7] and molecular orientations [8]. FPALM has been applied to a variety of biological imaging applications, including membrane, cytoskeletal, nuclear, and cytoplasmic proteins in fixed and living cells.

This tutorial describes how to successfully obtain and analyze FPALM images. Additionally, examples of successful biological applications and potential new applications of the technique will be described. The major topics to be covered are: i) Concepts of FPALM; ii) Instrumental setup (Fig. 1) and iii) Applications.

\section{References}

1. Hess ST, Gould TJ, Gudheti MV, Maas SA, Mills KD, et al. (2007) Dynamic clustered distribution of hemagglutinin resolved at $40 \mathrm{~nm}$ in living cell membranes discriminates between raft theories. Proc Natl Acad Sci U S A 104: 17370-17375.

2. Hess ST, Girirajan TP, Mason MD (2006) Ultra-high resolution imaging by fluorescence photoactivation localization microscopy. Biophys J 91: 4258-4272.

3. Gould TJ, Verkhusha VV, Hess ST (2009) Imaging biological structures with fluorescence photoactivation localization microscopy. Nat Protoc 4: 291-308. 
4. Bates M, Huang B, Dempsey GT, Zhuang X (2007) Multicolor super-resolution imaging with photo-switchable fluorescent probes. Science 317: 1749-1753.

5. Shroff H, Galbraith CG, Galbraith JA, White H, Gillette J, et al. (2007) Dual-color superresolution imaging of genetically expressed probes within individual adhesion complexes. Proc Natl Acad Sci U S A 104: 20308-20313.

6. Juette MF, Gould TJ, Lessard MD, Mlodzianoski MJ, Nagpure BS, et al. (2008) Threedimensional sub-100 nm resolution fluorescence microscopy of thick samples. Nat Methods 5: 527-529.

7. Huang B, Wang W, Bates M, Zhuang X (2008) Three-dimensional super-resolution imaging by stochastic optical reconstruction microscopy. Science 319: 810-813.

8. Gould TJ, Gunewardene MS, Gudheti MV, Verkhusha VV, Yin SR, et al. (2008) Nanoscale imaging of molecular positions and anisotropies. Nat Methods 5: 1027-1030.

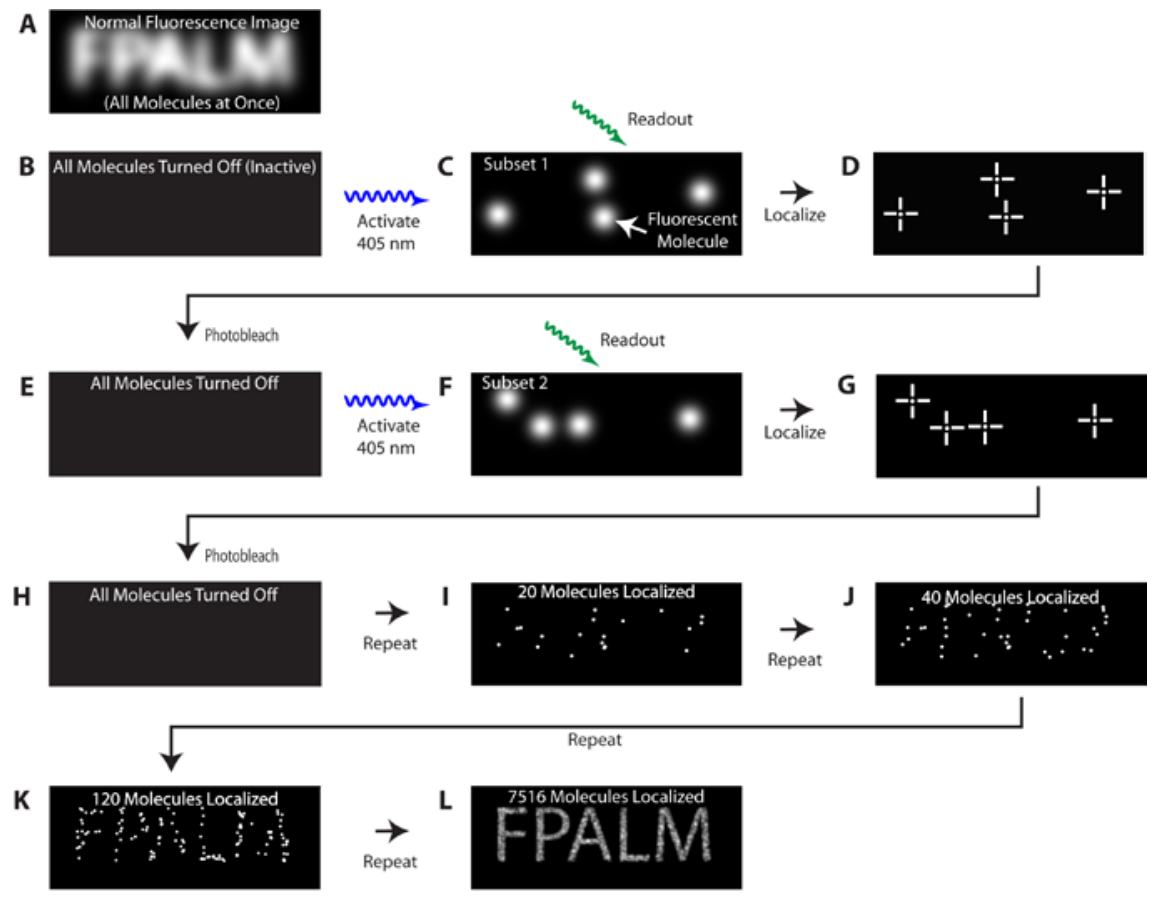

Figure 1. The molecules in the sample are initially inactive $(\mathrm{B}, \mathrm{E}, \mathrm{H})$ until an activation laser is used to active a subset of molecules in the sample $(\mathrm{C}, \mathrm{F})$. The activation laser is turned off and the fluorescence from the labeled proteins that were photoactivated is collected using a readout laser. Concurrently with the readout laser illumination, a high-sensitivity camera records movie frames of the fluorescence of the photoactivated molecules. These movie frames are analyzed to identify and localize activated molecules $(D, G, I-K)$ for the duration of their fluorescence. Molecules will spontaneously photobleach under the high-intensity illumination of the readout laser, which reduces the number of visible molecules to a low enough density that each visible molecule is distinct from others. The process of activation, imaging, localization, and photobleaching is repeated for many cycles to generate data containing tens or hundreds of thousands of molecules. Final images are rendered by plotting the positions of all localized molecules. 